\title{
BMJ Open "Caring About Me": a pilot framework to understand patient-centered care experience in integrated care - a qualitative study
}

\author{
Alaa Youssef (D , ${ }^{1,2}$ David Wiljer, ${ }^{2,3}$ Maria Mylopoulos, ${ }^{4}$ Robert Maunder, ${ }^{2,5}$ \\ Sanjeev Sockalingam ${ }^{2,6}$
}

To cite: Youssef A, Wiljer D, Mylopoulos M, et al. "Caring About Me": a pilot framework to understand patientcentered care experience in integrated care - a qualitative study. BMJ Open 2020;10:e034970. doi:10.1136/ bmjopen-2019-034970

- Prepublication history and additional material for this paper are available online. To view these files, please visit the journal online (http://dx.doi. org/10.1136/bmjopen-2019034970).

Received 15 0ctober 2019 Revised 21 April 2020 Accepted 18 June 2020

\section{ABSTRACT}

Objective The aim of this study is to examine patients' experiences in integrated care (IC) settings.

Design Qualitative study using semistructured interviews.

Settings Two IC sites in Toronto, Canada: (1) a community-based primary healthcare centre, supporting patients with hepatitis $\mathrm{C}$ and comorbid mental health and substance use issues; and (2) an integrated bariatric surgery programme, an academic tertiary care centre. Participants The study included patients $(n=12)$ with co-occurring mental and physical health conditions. Seven participants (58\%) were female and five (42\%) were male. Methods Twelve indepth semistructured interviews were conducted with a purposeful sample of patients $(n=12)$ with comorbid mental and physical conditions at two IC sites in Toronto between 2017 and 2018. Data were collected and analysed using grounded theory approach. Results Four themes emerged in our analysis reflecting patients' perspectives on patient-centred care experience in IC: (1) caring about me; (2) collaborating with me; (3) helping me understand and self-manage my care; and (4) personalising care to address my needs. Patients' experiences of care were primarily shaped by quality of relational interactions with IC team members. Positive interactions with IC team members led to enhanced patient access to care and fostered personalising care plans to address unique needs.

Conclusion This study adds to the literature on creating patient-centredness in IC settings by highlighting the importance of recognising patients' unique needs and the context of care for the specific patient population.

\section{INTRODUCTION}

Despite the significant attention and quality improvement efforts that followed the 'Crossing the Quality Chasm' report by the Institute of Medicine, notable gaps in care delivery persist for patients with complex care needs. ${ }^{1-4}$ Although individuals with complex care needs, defined as comorbid existing physical and mental health conditions, comprise a significant proportion of health service users, they tend to have worse health outcomes, poor care experiences and
Strengths and limitations of this study

- This study addresses an important gap in the literature on patient experience and presents a theoretical framework to systematically understand patients' experiences in integrated care.

- This study identifies four key care domains integral to patients perceiving patient-centredness.

- Generalisability of this framework to other care settings and context warrants further investigation given the small sample size of this study's population.

increased healthcare utilisation. ${ }^{45}$ Delivering high-quality care that improves individuals' experiences of care and the health of populations requires healthcare systems capable of adapting to a diverse range of patient needs, emerging multimorbidity and person-specific factors. ${ }^{467}$

Integrated care (IC) is a system-based care delivery model that evolved to bridge fragmentation in care delivery in primary care settings. ${ }^{8-12}$ Despite variation in IC implementation in care settings, ${ }^{13} 14$ the broader health system aims ${ }^{9}{ }^{10}$-improve population health outcomes, support cost-effectiveness and promote patient-centredness-are similar. ${ }^{1}$ Notwithstanding the extensive research supporting the effectiveness of IC to improve population health outcomes, it remains unclear how IC promotes patientcentred care experience from the patient's perspective.

While patient-centred care is a hallmark feature of high-quality care in IC, the construct is still in its infancy, with limited empirical and clinical evidence to indicate how this construct is conceptualised and operationalised in practice. For example, a robust conceptual framework that demarcates the principal care values that define patient-centred care experience is not well established. ${ }^{15}$ Moreover, 


\begin{tabular}{lllll}
\hline Table 1 & Demographic and clinical characteristics of participants in this study \\
\hline ID & Gender & Setting & Time in programme & Comorbidities ${ }^{*}$ \\
\hline 001 & F & BSP & 3years & Obesity-associated comorbidities, osteoarthritis, personality disorder. \\
002 & M & BSP & 5years & Obesity-associated comorbidities, depression. \\
003 & F & BSP & 8years & Obesity-associated comorbidities, MDD. \\
004 & F & BSP & 8years & Obesity-associated comorbidities, MDD, GAD. \\
005 & F & SRCHC & 1year & Hepatitis C, GAD, depression, alcohol abuse. \\
006 & M & SRCHC & 1year & Hepatitis C, osteoporosis, chronic pain, diabetes, GAD, MDD, PTSD, \\
& & & & ADHD, SA. \\
007 & F & SRCHC & 1year & Hepatitis C, depression, SA. \\
008 & M & SRCHC & 1year & Hepatitis C, depression, SA. \\
009 & F & BSP & 5years & Congenital hip dysplasia, MDD, alcohol abuse. \\
010 & F & BSP & 5years & Obesity-related comorbidities, alcohol abuse, bipolar disorder, BED. \\
011 & M & SRCHC & 6 months & Hepatitis C, depression. \\
012 & M & SRCHC & 1 year & Hepatitis C, HIV, depression.
\end{tabular}

*Obesity-associated comorbidities (including diabetes, sleep apnoea, hypertension), MDD, GAD, hepatitis C, PTSD, ADHD, addiction, SA, BED and HIV.

ADHD, attention deficit hyperactivity disorder; BED, binge eating disorder; BSP, Bariatric Surgery Program; F, female; GAD, generalised anxiety disorder; M, male; MDD, major depressive disorder; PTSD, post-traumatic stress disorder; SA, substance abuse; SRCHC, South Riverdale Community Health Centre-Hepatitis-C programme.

the lack of consensus in defining related key concepts, such as 'patient-centred care', 'patient experience' and 'patient satisfaction', has affected how these concepts are operationalised and assessed in practice. ${ }^{16-18}$ As a result, the absence of this empirical knowledge has limited our ability to reliably evaluate important care domains from the patient's perspective with respect to patient-clinician communication and relationship construction. ${ }^{19-24}$

This study sets out to examine patient-centred care experience from the perspective of patients with coexisting health conditions in IC settings. The aim is to elucidate essential care elements for a patient-centred care experience in IC to inform evaluation of patients' care experiences in IC.

\section{METHODS}

To examine how patients perceive patient-centred care experience in IC, this qualitative study used a constructivist grounded theory (GT) methodology. ${ }^{25}$ Constructivist GT is used to gain an indepth understanding of phenomena while recognising how social contexts, interactions, sharing viewpoints and interpretative analysis of patient and the researcher influence understanding. ${ }^{26} 27$ Semistructured interviews were used to examine the care experiences of patients with comorbid mental and physical conditions receiving care at two distinct IC sites in Toronto, Canada between 2017 and 2018 (table 1).

In this study, the two IC settings were identified as sites that would enable us to conduct cross-case analysis. The rationale for a cross-case analysis was to examine variations in patient-centred care experiences given differences in population care needs, contextual factors and the level of clinical setting integration. IC settings were informed by the Center for Integrated Health Solutions (CIHS) integration framework, where IC is defined as a continuum of care encompassing a range of care models that vary in structure primarily based on the degree of mental and physical health services integration, ranging from coordinated, co-located (collaborative care), to fully integrated care models (behavioural health integration)..$^{614}$ To examine the value of physical and behavioural health integration on patients' experiences, the Toronto Community Hepatitis C Program (TCHCP) at South Riverdale Community Health Centre (SRCHC) was identified as a community healthcare centre adopting an integrated behavioural health primary care model as described on the CIHS continuum of integration framework. The TCHCP supports patients managing hepatitis C, substance use and housing insecurity. ${ }^{28}$ The other IC setting was an academic-based medical centre, the Toronto Western Hospital Bariatric Surgery Program (TWH-BSP), a collaborative care bariatric surgery programme supporting patients with severe obesity and is ranked level 5 as per the CIHS continuum of integration. ${ }^{30} 31$ Therefore, collecting participant data from both of these two IC sites allowed us to explore nuances in patients' experiences among diverse patient groups with distinct care needs.

\section{Participants}

Our purposeful sample included patients with coexisting mental and physical illnesses so as to gain an insight into the complexity of self-management of chronic health conditions and the value of physical and behavioural health integration from the patient's perspective. ${ }^{32} \mathrm{We}$ focused on patients with two or more comorbid conditions as a common source of complexity according to the 
literature on the chronic care model and IC (table 1). ${ }^{11}$ Patients at both sampling sites were eligible for participating if they had two or more physical and mental health comorbidities and have been receiving care at their respective IC setting for at least 3-6 months. We used semistructured individual interviews to facilitate candid disclosure of personal experiences. We conducted a total of 12 indepth semistructured interviews and had 6 interviews per site. Following the GT logic, sample size was not determined a prior but rather informed by the iterative process of data collection and analysis. For example, in this study initial sampling was exploratory and provided the interviewer (AY) with a point of departure that gradually developed to concrete categories with iterative coding and memo writing. ${ }^{33}$ Sampling continued until theoretical saturation was achieved, defined as the point where further interviews did not advance the conceptual depth of the developed categories or reveal new dimensions of the relationship among categories. ${ }^{27} 3334$ Participants were recruited by phone or email by a study researcher (AY) and received a compensation of $\$ 20$ as a token of appreciation for participating in the study.

\section{Data collection}

Primary interview questions were informed by collaborative care core principles (ie, patient-centred care, teambased care, measurement guided and population-based care) and focused on patients' experiences accessing and interacting with care team members in IC settings. ${ }^{12} 3536$ Initial interview questions were open-ended and developed iteratively with the research team (online supplementary appendix 1). Subsequent revisions of the interview guide were informed by emerging themes and sensitising concepts generated through data collection and analysis. In this study, sensitising concepts referred to relevant concepts that facilitated exploration of new ideas and critical analysis of the data. ${ }^{27}$ We revised the interview guide questions informed by results from data analysis as to iteratively challenge, refine and elaborate on the emerging themes.

Interviews lasted approximately $90 \mathrm{~min}$ and were facilitated by a trained researcher (AY), a PhD candidate, who received formal training in qualitative research methodology. The length of each interview was determined by the patient's level of comfort disclosing their perceptions and sharing their experiences. We completed a total of 12 interviews resulting in $1080 \mathrm{~min}$ of recordings that were used for data analysis. All participants provided informed consent for the interviews to be audiotaped and professionally transcribed.

\section{Patient and public involvement}

Patients from the examined settings informed interview guide development and purposeful participant selection to explore emerging themes. Members from the IC teams at both sites verified study findings and finalised the manuscript. We communicated the research findings to patients and the public through poster and oral presentation at relevant events.

\section{Data analysis}

We used a constant comparative approach to simultaneously collect and analyse data. Analysis of interview transcripts was iterative and inductively driven, using line-by-line coding, open coding, focused coding and axial coding, to abstract emerging concepts that informed framework construction (online supplementary appendix 2). This analytical approach enabled exploration of emerging themes, contrast experiences within and across sites, impose new questions, and refine developing theory. Through the data collection and analysis process, the researcher (AY) independently coded the data from an exploratory lens and generated a code book. By comparing experiences, views, situations and contexts from the same and different individuals, the researcher (AY) started identifying emerging themes and gradually refined the coding schema. Furthermore, iterative and biweekly discussions with the research team (MM and SS) allowed for triangulation of the data from multiple perspectives. Research team (DW, RM, MM and SS) discussions inspired questions to help evaluate emerging hypotheses, develop theoretical categories and identify constructs that formulated the thematic framework of how patients conceptualised a patient-centred care experience.

Throughout the study, the researcher (AY) incorporated memo writing to reflect on individual cases, interview settings, participants' responses, emerging concepts and assess preconceived notions (online supplementary appendix 3). The researcher maintained an audit trail of the analysed interviews, memo writings and team discussions. The final stages of the analysis used the NVivo software to conduct cross-case analysis to identify patterns and variations in codes across cases. It also served as a tool to visualise and examine the development of a thematic framework.

\section{RESULTS}

Analysis of patient interviews revealed that patient-centred care experience in IC settings is dynamic and evolving (figure 1). Four interconnected themes explained this dynamic process from the patient's perspective. In our analysis, 'Caring About Me' emerged as the overarching theme describing core care values linked to patients' interactions with the IC teams. The three additional themes, 'Collaborating with Me', 'Sharing Knowledge and Developing a Monitoring Self' and 'Personalising Care to Address My Needs', worked in service of this central theme. The following sections describe these four themes in further detail.

\section{Theme 1: 'Caring About Me'}

Patients reflected on their personal interactions with the care team and perceived the care team to be genuinely 


\section{Caring about Me}
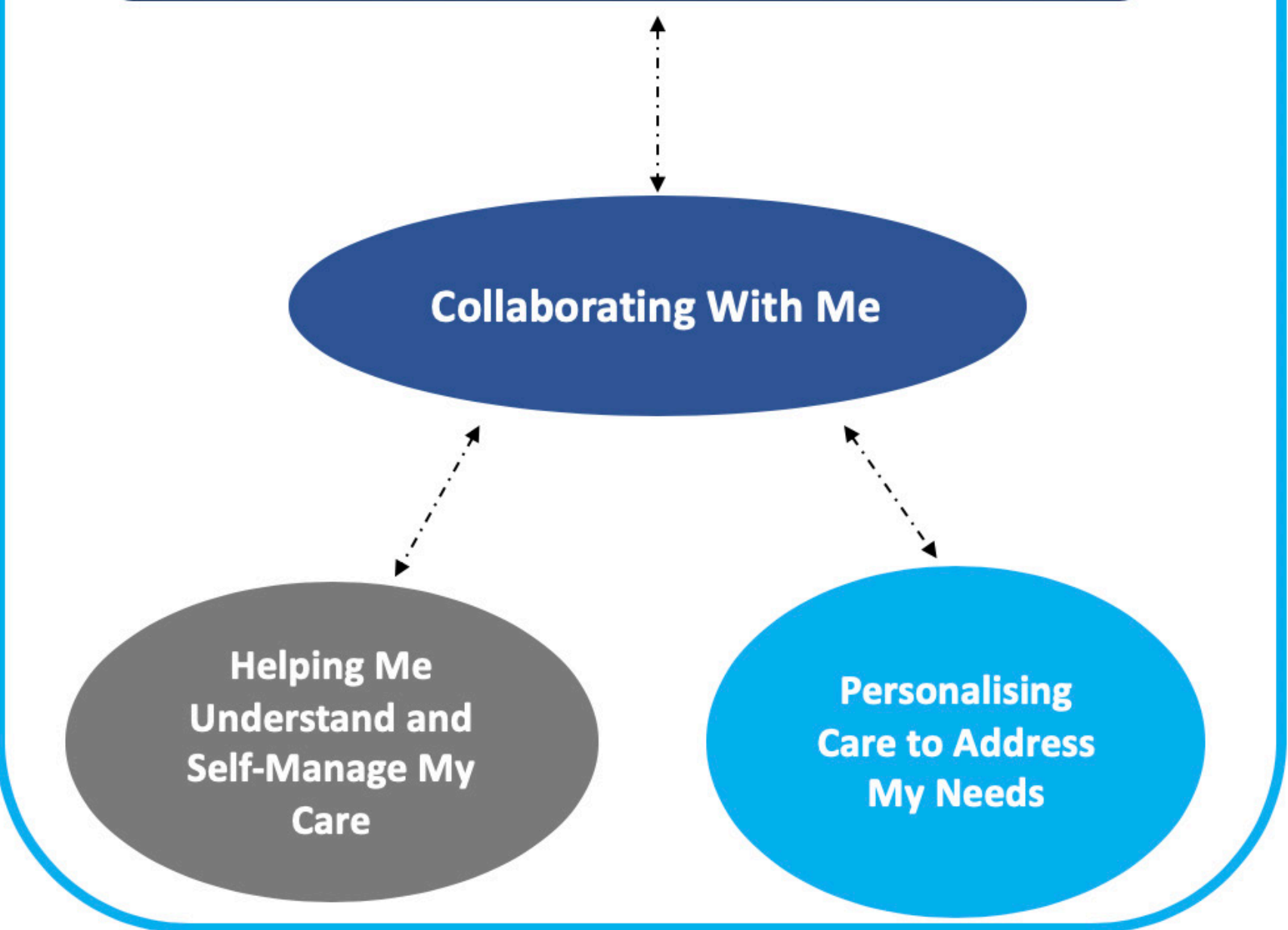

Figure 1 'Caring About Me': a framework to understand patients' experiences in integrated care settings.

caring about them despite variations in contexts, conditions and demographics. Participants across sites shared similar experiences where they described being at the centre of care of their provider/care team. Attributes linked to the 'Caring About Me' theme described the constructive nature of patient-care team interactions in IC that helped patients express their care needs, normalise failure and develop entrusted longitudinal relationship with their care team members.

A defining component of patient-centred conversations was helping patients recognise their care needs and express their preferences. Participants across sites valued clinicians' capabilities in recognising patients' needs and helping them address their care preferences during both illness and wellness. Participants highlighted the shift in care needs at these transitions between illness and wellness moments. For example, participants identified lacking the capacity to articulate their needs and preferences at times of illness. Participants also reported greater confidence in their care team's knowledge and ability to address their care needs when their team framed their discussions in a way that empowered them to understand and manage their physical and emotional needs at vulnerable times.

One participant described feeling vulnerable recovering from bariatric surgery complications. Reflecting on how her physical weakness affected her capacity to recognise her care needs, the participant praised her care team's determination in helping her overcome feelings of disappointment and her lack of motivation in completing the recommended rehabilitation exercise.

Then there were some the physio nurses that were helping. And then there was another nurse who was kind of like a, get out of bed, you're going to get out of bed, you're going to sit in this chair, you're going to...And I didn't like it, but I would praise her now to say thank you. (BSP, case 004) 
Conversely, at times of wellness, the patient-physician dialogue focused on patients' concerns and co-constructing care plans. For example, some participants reported discussing research regarding new treatment options with their primary care provider (PCP). This process enabled patients to gain autonomy while sharing with their care providers the responsibility for their care.

My doctor obviously does her research. She follows up. She actively listens, and again I have to say she follows up. If not right away, she'll follow up via an alternate appointment or via email. So, if she doesn't have an answer for me right away, she gets that answer for me once she does her research or figures it out. (BSP, case 002)

Feeling respected and accepted was a defining feature of patients' experiences in IC. The care team's nonjudgemental approach and respect of each patient's journey enabled patients to perceive care settings as open spaces, where they could share their personal values, preferences and express their needs without feeling judged. Participants reported that their own negative perceptions about themselves secondary to their illness sometimes served as a potential barrier to seeking help. Through the IC team's non-judgemental and accepting approach, patients felt this helped correct their negative self-perception and increased their trust of their care providers.

And then the psychiatrist kind of says, okay, so this is how I want you to kind of look at things, or this is a perspective that I want you to think about as I journey for the next week or two. You know, I came in today, and I said, you know, I failed over the last two weeks, I stopped taking my medication. And he immediately said, I wouldn't use the term failure. You've had a setback, you know. And he's like, you know, we all have setbacks in our journey of recovery, it's very common. So, you didn't fail, you're not a failure at all. Like, that's his response. He's an amazing clinician, he's a great doctor. (BSP, case 010)

\section{Theme 2: collaborating with me}

Patients reported a stronger sense of alliance with the patient-care team within the IC settings. Patient alliance with the care team was fostered by supporting patientaccess to timely care, advocating for patients' concerns within the care team ('being my voice'), connecting patients to support resources or promoting patient engagement in a safe and open environment. Patients sought care team collaborations during periods of setbacks and complications by mobilising their care team to provide immediate attention or prompt access to specialty care.

For example, TWH-BSP patients indicated that their PCP grounded them during periods of distress or when they lacked information to feel confident in managing their physical and emotional care needs. In this context, IC systems facilitated patients' immediate access to their
PCP, where patients felt supported during setbacks, learnt about accessible support services and accessed specialty services:

It feels like, [nurse], you're not the only one, it's okay. We have supports, like, we have systems in place to support you. Like, she just helped ground me to know that, you know what, you're going to be okay. Like, it was amazing. And then she was just right on it, she was so professional. Like, within a week I had an appointment to see the addiction specialist. I think that's amazing, like that's amazing care. (BSP, case 010)

Furthermore, while most patients aspired to gain autonomy for their care, some patients required an advocate to convey their care needs and to navigate the healthcare system to address their needs. IC was identified as a gateway for patients to find 'a voice' that they could trust to express their needs more confidently to the care team and to leverage system resources. For example, a participant recounted lacking the capacity to advocate for herself and having anxiety with undergoing revisional surgery at the same hospital where their original bariatric surgery was conducted. A distinguishing feature of IC teams working with patients with complex comorbid illness was the ability to recognise patients' unexpressed needs and become an additional 'voice' advocating for patients and connecting them to necessary care services:

I mean, I wasn't standing there when she did it, but from what I understand, I was here, and she walked out to the hall. She gathered the team together and she said, this girl is not going back to [hospital X], we are going to look after her, we need a doctor. And that's how I got my help.... I think, at that time, I really just focused on the dietician. She was my connecter at that point...I think it was just that she was my voice. She was a voice that people listened to. (BSP, case 004)

Theme 3: sharing knowledge and developing a monitoring self Participants' experiences in IC settings revealed how sharing their experience and knowledge with other patients, such as in support groups, provided a space for patients to share the ways that physical and mental illness (obesity, surgery, hepatitis C, depression) influenced their lives. Finding commonalities in their experiences allowed them to question assumptions about their thinking, feelings and habits, to care for themselves. This process of sharing knowledge and experiences was facilitated by healthcare providers (ie, formally facilitating support groups), who enabled patients to develop their coping skills and cultivate the capacity to self-manage their health and well-being.

In addition, patients' discussions with care providers encouraged them to share their challenges, seek knowledge, gain confidence and develop coping skills to manage their symptoms better and improve their health 
outcomes. For example, patients perceived these discussions as an opportunity for them to build rapport with their care providers and feel connected and supported.

A participant highlighted the importance of this process of knowledge sharing as a means to strengthen the patient-care provider alliance:

The dietician you see her most. There, the dieticians, their level of knowledge across the board was phenomenal, so that's what I appreciated. And we developed a rapport. When you develop a rapport with anybody, it always makes things easier. (BSP, case 009)

Similarly, patients experienced both individual and group-based care knowledge-sharing interactions as crucial care elements in helping them understand the need for care services and feeling more confident in engaging in preventative and active treatments to improve their overall health.

So, she's patient with me, she will explain stuff to me so that I can do it, like on the weekend I had to do the bandage on my own, so she showed me how to do it. It's an amazing place with amazing people. (SRCHC, case 008)

\section{Theme 4: personalising care to address patients' unique needs}

Patients identified their varied and individual care needs and highlighted how important it was to tailor treatments to address these unique care needs in order to improve their health outcomes. For instance, the complexity of obesity-related diseases in the bariatric surgery patient population contributes to surgical complications in some individuals. While managing physical and emotional shifts during this acute stage is a well-recognised challenge, patients felt well cared for by physicians, nurses and other team members, who listened and invested time in understanding their whole story to address their unique care needs during their treatment journey.

And my surgeon, Dr. $\mathrm{X}$ has performed four surgeries on me, so I know her well and I email with her and she asks for feedback as well, so I think that...And she cares, Dr. X, she cares, and she sits, and she listens, and she tries to figure things out, and then when things aren't going great, like I've had...Actually, I had one surgery where I was just getting untangled, basically, and I said, I'm adopted, and I said I found out that colon cancer runs in my family so when you're doing this is there any way you could check things out? She did, she ran my colon and found a tumor and it was removed last year, and benign, so that was great. (BSP, case 003)

Patients mentioned the challenges of self-managing their chronic conditions, seeking help and adhering to their treatment plans as a result of psychosocial factors. Specific psychosocial factors reported by patients included depression and substance use issues, which interfered with care seeking and ability to manage their chronic health issues, specifically obesity and hepatitis C.

I do have depression and I am back on medication and that kind of...Actually, when we're speaking of weight gain, I had three surgeries last year on my bowels, and I couldn't run for a long time, and I got depressed, and I started eating again, and I gained quite a bit of weight that I'm still trying to take off. And, yeah, the weight gain and depression, for me, do go hand-in-hand. (BSP, case 003)

Importantly, participants' interviews highlighted the importance of IC clinicians recognising the patient's whole situation, particularly during vulnerable times when individuals might not fully understand or recognise the impact of illness on various domains of their life. This process of shared deliberation between the clinician and the patient in IC was key in addressing the varied needs of patients and helping patients realise the impact of their illness on their social, work and functional life.

Yeah, I mean, he's taking a vested interest in my whole story. It's not just about prescribing medication and booking a follow-up appointment, checking for sideeffects, no. It's about the whole story, like what's going on in your life. Like, for instance, today we were talking about me going into a treatment program. You know, I'm not going to get teary, but it really touched me...He asked me, what about work, what about your work situation. Because he wants to know, if you want to do a treatment program, you know, are you able to take the time off work, are you going to be supported at work, are you going to be able to afford it. Like, he cares, you know. He's recognizing my whole situation, my whole story. Like, that means a lot to me. (BSP, case 010)

A participant recounted his experience being helped and receiving care from their PCP in a community-based IC setting after suffering an acute physical trauma. The patient had a history of care avoidance due to prior difficult experiences with care providers in acute care settings. As a result, he placed his trust in his PCP in the IC programme to address these complex physical issues.

Yeah. And the car accident was last year. My ear was dangling from the front here, it was off, and I cannot hear on that side no more. I had five broken ribs, I had a dislocated shoulder, I had multiple wounds on my hands like cuts and stuff that needed injury. Yeah. So, she stitched me up and then she gave me the stuff I needed because usually I just do all those things myself. (SRCHC, case 008)

Patients reported similar examples where care providers in IC settings used a holistic approach that was able to adapt to patients' unique care needs and overcome psychosocial barriers to care, such as anxiety, stigma and difficulty trusting healthcare providers due to past relational trauma. 


\section{DISCUSSION AND CONCLUSION}

\section{Discussion}

The purpose of this study was to bridge the theorypractice gap on patient-centred care experience in IC settings. Despite the popularity of 'patient-centred care' as a distinguished attribute of high-quality care, limited empirical and clinical evidence indicates how this construct is conceptualised and operationalised in practice. Using a grounded theory approach to develop our theoretical understanding of the patient's perspective, this study proposes the 'Caring About Me' framework, which demarcates key features of patient-centred care experience in IC settings (figure 1).

The quality of patients' interactions with the care team was a defining element of patient-centred care experiences in IC settings, regardless of the complexity of patient care needs. Based on our data, participants perceived their care to be patient-centred if they felt supported, listened to, respected, accepted, and their care needs and preferences were recognised and reviewed through collaborative discussions. Moreover, patients' perceptions of their IC experience developed incrementally through their longitudinal interactions with care team members.

Patients' perception of effective care in IC settings was strongly influenced by perceived patient-care team interactions, specifically the ability of IC teams to recognise patients' care needs and establish entrusting relationships. The cultivation of these entrusted relationships was distinct to the care sites. For example, patients' experience within SRCHC involved a strong sense of feeling accepted, mutual respect and a non-judgemental approach, which was supported by the creation of a safe, 'open space'. In contrast, TWH-BSP focused on recognising the complexity and uniqueness of obesity-related comorbidities and how these factors impacted TWHBSP individuals' quality of life, which fostered trustable patient-care team relationships within this setting. These findings underscore that it is important for IC sites to consider patients' needs, context and values to enable patients' experience of care-centredness in IC.

In addition to patients' interaction with the IC team, patients' complexity and variations in their care needs influenced how this 'Caring About Me' model addressed patients' specific needs. For example, at SRCHC programme, patients' care needs demanded supporting chronic disease management (primarily hepatitis C care), addressing social disparities and promoting behavioural change through health education. The programme met these care needs through the creation of an open and inclusive space that engaged patients in support groups where they could share their experiences with one another, gain further awareness and engage in and learn further self-management skills for hepatitis C. Conversely, at the TWH-BSP, patient-centredness unfolded through patient-care team interactions at an individual level throughout the preparation for and postsurgical follow-up, which recognised each patient's individual journey and the multiple factors influencing obesity care.
In both settings, patient-care team alliance was fostered by the IC team adapting their treatment approach within each setting to accommodate the variability in patients' care needs.

Overall, findings from this study align with the empirical literature on patient-centred care. Previous work by Kvåle and Bondevik and Marshall et $a l^{378}$ identified the importance of patients feeling respected, connected and involved in care planning and decision-making in acute care settings, similar to the 'Caring About Me' theme in this study. Importantly, this study advances our understanding of the patient-centredness phenomenon by providing insights into how patients perceive patientcentred care in IC. Specifically, this study highlights that patient-centred care experience is an evolving process that develops through productive patient-clinician interactions. In the IC context, these productive interactions flourished as the care team amended their treatment approach to align with the recognised patient population care needs and context. Building a strong treatment alliance was vital for patients to have a longitudinal relationship with their PCPs.

\section{Limitations}

Notwithstanding the inherent limitations to generalising conclusions from this study, the purpose of this GT study was to advance our understanding of patient-centred care experience in IC settings and not to produce generalisable findings. Future studies should investigate the universality and applicability of this empirical model to other care delivery models and populations. Although our sample size may be perceived as a limitation, we attempted to minimise selection bias to a specific site or population by exploring this phenomenon across multiple sites in parallel and throughout patients' care journey within IC. Recognising that researchers' position and perspectives inevitably influence access to findings and knowledge construction, adopting constructivist GT methodology affords strategies that helped account for these limitations and assert research rigour. ${ }^{26}{ }^{27}$ These strategies include contrasting participants' account within and across cases and situations, enabling triangulating data from multiple perspectives, and establishing researcher reflexivity through memo writing and questioning one's preconceived notions and meta-position while constructing the emerging theory.

\section{Conclusion}

This study generated the 'Caring About Me' framework that describes patient-centred care experience from the patient's perspectives. This model identified the core constructs underpinning the process of patientcentredness in IC. Our findings indicated that the versatility of the IC team to amend their care processes, to the context and patient population care needs, was critical to facilitating patient-centred care experience. This model needs further testing, validation and development in different contexts. 
The "Caring About Me" framework provides a practical means to understanding how "patient-centred care" may be practiced in reality. Findings from this study offer a theoretical foundation to inform the utilisation of patient-centred quality measures that better capture valuable quality of care domains that align with patient expectations. Developing this body of practice-based evidence is critical to advancing the implementation of evidencebased research to practice. ${ }^{39-41}$ Future studies could advance this model by exploring the external facilitators and barriers to promoting patient-centredness from the care-team's perspective.

\section{Author affiliations}

${ }^{1}$ Institute of Medical Science (IMS), University of Toronto, Toronto, Ontario, Canada ${ }^{2}$ Psychiatry, Faculty of Medicine, University of Toronto, Toronto, Ontario, Canada ${ }^{3}$ Education, Technology \& Innovation, UHN Digital, University Health Network, Toronto, Ontario, Canada

${ }^{4}$ Wilson Centre, Undergraduate Medical Professions Education and Department of Paediatrics, University of Toronto, Toronto, Ontario, Canada

${ }^{5}$ Psychiatry, Sinai Health System, Toronto, Ontario, Canada

${ }^{6}$ Education, Centre for Addiction and Mental Health, Toronto, Ontario, Canada

Acknowledgements The authors would like to thank all participants and clinical facilitators at the Toronto Community Hepatitis C Program (TCHCP) at South Riverdale Community Health Centre (SRCHC) and the Toronto Western Hospital Bariatric Surgery Program (TWH-BSP) who participated and supported recruitment for this study.

Contributors AY was responsible for data collection, transcript analysis and manuscript drafting. AY, DW, MM, RM and SS contributed to study design, iterative data analysis, manuscript drafting and review.

Funding This work is supported in part by the Medical Psychiatry Alliance, a collaborative health partnership of the University of Toronto, Centre for Addiction and Mental Health, Hospital for Sick Children, Trillium Health Partners, Ontario Ministry of Health and Long-Term Care, and an anonymous donor.

Competing interests None declared.

Patient consent for publication Not required.

Ethics approval This study was approved by the University Health Network (UHN) Research Ethics Board.

Provenance and peer review Not commissioned; externally peer reviewed.

Data availability statement All data relevant to the study are included in the article or uploaded as supplementary information.

Open access This is an open access article distributed in accordance with the Creative Commons Attribution Non Commercial (CC BY-NC 4.0) license, which permits others to distribute, remix, adapt, build upon this work non-commercially, and license their derivative works on different terms, provided the original work is properly cited, appropriate credit is given, any changes made indicated, and the use is non-commercial. See: http://creativecommons.org/licenses/by-nc/4.0/.

ORCID iD

Alaa Youssef http://orcid.org/0000-0001-6505-8236

\section{REFERENCES}

1 Institute of Medicine. Crossing the quality chasm : a new health system for the 21 st century. Washington, D.C: National Academy Press, 2001.

2 Dentzer S. Still crossing the quality chasm--or suspended over it? Health Aff 2011;30:554-5.

3 Fernandopulle R, Ferris T, Epstein A, et al. A research agenda for bridging the 'quality chasm'. Health Aff 2003;22:178-90.

4 Vogeli C, Shields AE, Lee TA, et al. Multiple chronic conditions: prevalence, health consequences, and implications for quality, care management, and costs. J Gen Intern Med 2007;22:391-5.
5 Ludman EJ, Katon W, Russo J, et al. Depression and diabetes symptom burden. Gen Hosp Psychiatry 2004;26:430-6.

6 Peek CJ, Baird MA, Coleman E. Primary care for patient complexity, not only disease. Fam Syst Health 2009;27:287-302.

7 Safford MM. The complexity of complex patients. J Gen Intern Med 2015;30:1724-5.

8 Bauer AM, Thielke SM, Katon W, et al. Aligning health information technologies with effective service delivery models to improve chronic disease care. Prev Med 2014;66:167-72.

9 Berwick DM, Nolan TW, Whittington J. The triple aim: care, health, and cost. Health Aff 2008;27:759-69.

10 Sikka R, Morath JM, Leape L. The quadruple aim: care, health, cost and meaning in work. BMJ Qual Saf 2015;24:608-10.

11 Chwastiak L, Vanderlip E, Katon W. Treating complexity: collaborative care for multiple chronic conditions. Int Rev Psychiatry 2014;26:638-47.

12 Chwastiak LA, Jackson SL, Russo J, et al. A collaborative care team to integrate behavioral health care and treatment of poorly-controlled type 2 diabetes in an urban safety net primary care clinic. Gen Hosp Psychiatry 2017;44:10-15.

13 Peek CJ. Executive Summary-Lexicon for behavioral health and primary care integration: concepts and definitions developed by expert consensus. Rockville, MD: AHRQ, 2013.

14 Heath B WRP, Reynolds K. A review and proposed standard framework for levels of integrated healthcare. Washington, D.C: SAMHSA-HRSA Center for Integrated Health Solutions 2013.

15 Stewart M. Towards a global definition of patient centred care. BMJ 2001;322:444-5

16 Weaver M, Patrick DL, Markson LE, et al. Issues in the measurement of satisfaction with treatment. Am J Manag Care 1997;3:579-94.

17 Murray CJ, Kawabata K, Valentine N. People's experience versus people's

18 van Campen C, Sixma H, Friele RD, et al. Quality of care and patient satisfaction: a review of measuring instruments. Med Care Res Rev 1995;52:109-33.

19 Epstein RM, Street RL. The values and value of patient-centered care. Ann Fam Med 2011;9:100-3.

20 Roseman D, Osborne-Stafsnes J, Amy CH, et al. Early lessons from four 'aligning forces for quality' communities bolster the case for patient-centered care. Health Aff 2013;32:232-41.

21 Campbell SM, Roland MO, Buetow SA. Defining quality of care. Soc Sci Med 2000;51:1611-25.

22 Donabedian $\mathrm{A}$. The quality of care. how can it be assessed? JAMA 1988;260:1743-8.

23 Donabedian A. Evaluating the quality of medical care. Milbank Mem Fund Q 1966;44:166-206.

24 Sunderji N, Ion A, Ghavam-Rassoul A, et al. Evaluating the implementation of integrated mental health care: a systematic review to guide the development of quality measures. Psychiatr Serv 2017;68:891-8

25 Charmaz K. Constructing grounded theory. London; Thousand Oaks, Calif: Sage, 2014.

26 Charmaz K. Grounded theory. London: SAGE Publications Ltd, 1995.

27 Charmaz K. Constructionism and the grounded theory method. In: Handbook of constructionist research, 2008: 397-412.

28 Mason K, Dodd Z, Sockalingam S, et al. Beyond viral response: a prospective evaluation of a community-based, multi-disciplinary, peer-driven model of HCV treatment and support. Int J Drug Policy 2015;26:1007-13.

29 Sockalingam S, Blank D, Banga CA, et al. A novel program for treating patients with trimorbidity: hepatitis $\mathrm{C}$, serious mental illness, and active substance use. Eur J Gastroenterol Hepatol 2013;25:1377-84.

30 Santiago VA, Warwick K, Ratnakumarasuriyar S, et al. Evaluation of a patient-care planning intervention to improve appointment attendance by adults after bariatric surgery. Can J Diabetes 2019;43:59-66.

31 Sockalingam S, Hawa R, Wnuk S, et al. Psychosocial predictors of quality of life and weight loss two years after bariatric surgery: results from the Toronto Bari-PSYCH study. Gen Hosp Psychiatry 2017;47:7-13.

32 Palinkas LA, Horwitz SM, Green CA, et al. Purposeful sampling for qualitative data collection and analysis in mixed method implementation research. Adm Policy Ment Health 2015;42:533-44.

33 Charmaz K. Constructing grounded theory: a practical guide through qualitative analysis. 2 edn. London: SAGE, 2014. 
34 Nelson J. Using conceptual depth criteria: addressing the challenge of reaching saturation in qualitative research. Qual Res 2017;17:554-70.

35 Katon WJ, Lin EHB, Von Korff M, et al. Collaborative care for patients with depression and chronic illnesses. $N$ Engl J Med 2010;363:2611-20.

36 Medicine AoP, Association AP. Dissemination of integrated care within adult primary care settings: the collaborative care model, 2016.

37 Kvåle K, Bondevik M. What is important for patient centred care? A qualitative study about the perceptions of patients with cancer. Scand J Caring Sci 2008;22:582-9.
38 Marshall A, Kitson A, Zeitz K. Patients' views of patient-centred care: a phenomenological case study in one surgical unit. $J$ Adv Nurs 2012;68:2664-73.

39 Green LW, Glasgow RE. Evaluating the relevance, generalization, and applicability of research: issues in external validation and translation methodology. Eval Health Prof 2006;29:126-53.

40 Glasgow RE, Green LW, Klesges LM, et al. External validity: we need to do more. Ann Behav Med 2006;31:105-8.

41 Cohen DJ, Crabtree BF, Etz RS, et al. Fidelity versus flexibility: translating evidence-based research into practice. Am J Prev Med 2008;35:\$381-9. 